\title{
Modélisation et essais cliniques en pédiatrie
}

\author{
Philippe Jacqmin, ${ }^{1}$ Nathalie Labouret, ${ }^{2}$ François Gueyffier ${ }^{3}$ et les participants à la Table Ronde \\ $n^{\circ} 6$ de Giens XX \\ 1 Société Exprimo, Beerse, Belgium \\ 2 Agence française de sécurité sanitaire des produits de santé (Afssaps), Saint-Denis, France \\ 3 Centre d'Investigation Clinique, Lyon, France
}

\section{Résumé}

Les essais cliniques sont plus difficiles à réaliser chez l'enfant, mais ils sont encore plus nécessaires que chez l'adulte : leur rareté constitue un véritable scandale éthique. La modélisation est une représentation fondée sur la logique et les mathématiques, applicable aux mécanismes des maladies et des médicaments. La simulation permet de manipuler des objets comme les essais cliniques. La prédiction de résultats simulés permet l'optimisation du plan expérimental et du plan d'analyse. La validation est une étape cruciale de la modélisation et de la simulation. Les participants de la Table Ronde recommandent : (i) d'envisager systématiquement le recours à la modélisation ; (ii) d'impliquer toutes les compétences requises, à toutes les étapes ; (iii) que toutes les données soient rendues accessibles ; (iv) que les cliniciens soient formés ; (v) que les spécialistes mettent au point des kits de formation; (vi) que les universités proposent une formation adéquate.

Mots clés : modélisation, simulation, essais cliniques, pédiatrie
La modélisation et la simulation $(\mathrm{M} \& \mathrm{~S})$ constituent une méthodologie adaptée à toutes les phases du développement du médicament. Le Comité d'Organisation des Tables Rondes de Giens a estimé que la pharmacologie clinique devait investir davantage les possibilités offertes par cette méthodologie. En 2003, une Table Ronde a fait le point sur les concepts de base de la M\&S. En 2004, une autre Table Ronde a envisagé les enjeux de l'application de la M\&S à la pédiatrie. Ce document s'efforce de retranscrire l'essentiel des débats consacrés au thème «modélisation et essais cliniques en pédiatrie » pendant les rencontres de pharmacologie de Giens 2004. Durant 3 jours, (i) les besoins en essais cliniques spécifiquement pédiatriques, ainsi que les obstacles rencontrés, ont été abordés du point de vue du régulateur, de l'industriel et du praticien ; (ii) un tour d'horizon des applications pédiatriques de la modélisation a été proposé à partir d'exemples ; (iii) l'intérêt des techniques de simulation d'essais cliniques fondées sur la modélisation a été illustré par l'optimisation de la planification des essais ; enfin, (iv) les aspects concernant la validation, les règles de bonne pratique, la formation et l'environnement professionnel ont été débattus dans l'optique de proposer des recommandations.

\section{Aperçu des besoins perçus par le régulateur, l'industriel et le praticien}

Encore de nos jours, les soins en pédiatrie se caractérisent par l'indigence de la pharmacologie clinique et du développement du médicament qui lui sont consacrés : les données sur les bases mêmes de la pharmacologie, incluant la pharmacocinétique, la pharmacodynamique et la sécurité, restent éparses et dans l'ensemble absentes. La fabrication des médicaments ne répond pas aux besoins spécifiques des enfants.

L'idée que l'enfant est un adulte en miniature a pu paraître attractive, notamment en ce qu'elle répond aux principes de simplicité et de parcimonie si importants pour la pratique efficace de la modélisation. Cependant, cette idée simple est totalement erronée et source de graves errements : (i) l'absorption, la distribution, le métabolisme et l'excrétion se distinguent plus par leurs différences que leur ressemblance avec ce qui est observé chez l'adulte, d'autant que ces phénomènes évoluent et présentent des changements drastiques à plusieurs étapes de la maturation infantile ; (ii) les maladies présentent souvent des particularités essentielles, quand elles ne sont pas totalement spécifiques de la population pédiatrique, concernant notamment les tumeurs, les infections, les affections neurologiques (déficit de l'attention, épilepsie, etc.) ; (iii) les effets des médicaments présentent des différences d'intensité non explicables par les seules particularités pharmacocinétiques, mais en relation avec les processus de maturation des organes et des systèmes, comme par exemple pour le cour, le cerveau, les dents, etc.

La régulation aux Etats-Unis d'Amérique a progressivement

\footnotetext{
* Pour la liste des participants, voir en fin d'article.
} 
influencé les stratégies de Recherche et Développement (R\&D) des industriels du médicament impliqués en pédiatrie, par l'encouragement (Paediatric Exclusivity en 1997, ${ }^{[1]}$ suivie par une augmentation significative du nombre d'essais cliniques chez l'enfant) mais aussi par la contrainte (Paediatric Rule en $1998,{ }^{[2]}$ Best Pharmaceuticals for Children Act $[\mathrm{BPCA}]^{[3]}$ en 2001 et Paediatric Equity Act en $2003^{[4]}$ ).

En Europe, à la suite de différentes actions de groupes de pression au rang desquels les rapports de précédentes Tables Rondes de Giens, la commercialisation de médicaments pour les maladies rares a été favorisée, par exemple en allégeant les dossiers de soumission, en fournissant des réponses plus rapides, ou en étendant la protection commerciale. De nouveaux développements applicables en 2006 concernent l'utilisation des médicaments en pédiatrie, une évaluation focalisée sur les besoins, l'examen des demandes par un Comité ad hoc de l'EMEA (European Agency for The Evaluation of Medicinal Products), un soutien scientifique et des recommandations pour le développement des médicaments dans les populations pédiatriques. ${ }^{[5]}$

\section{Enjeux et difficultés des essais cliniques en pédiatrie}

Les difficultés pour la conduite des essais cliniques pédiatriques s'expliquent par (i) une régulation particulière, justifiée par la nécessaire protection d'individus particulièrement vulnérables - par exemple, il n'est pas permis de conduire des essais sur volontaires sains en pédiatrie ; (ii) les tailles de population sont souvent réduites, d'autant qu'il est souvent nécessaire d'étudier séparément différentes classes d'âge ; (iii) le nombre et le volume des prélèvements sanguins sont par essence plus limités ; (iv) la prévention des douleurs et de l'anxiété revêt une importance toute particulière dans le contexte pédiatrique.

Ces difficultés reconnues et admises, la réalisation d'essais cliniques est pourtant encore plus cruciale chez l'enfant, en raison de l'importance des prescriptions hors autorisation de mise sur le marché (AMM), qui représentent $56 \%$ des prescriptions hospitalières, ${ }^{[6]}$ du défaut de préparations pharmaceutiques adaptées (entre 50 et $90 \%$ des médicaments prescrits aux enfants en Europe), ${ }^{[7]}$ et de l'absence d'information sur les spécificités pharmacocinétiques, d'efficacité et de sécurité. Les populations pédiatriques représentent autour de 100 millions d'individus dans l'Europe élargie. Le plus souvent, les pédiatres ont le choix entre une prescription hors AMM (au risque non documenté d'effets indésirables potentiellement mortels, ou d'inefficacité), et l'abstention alors que des médicaments potentiellement utiles sont disponibles avec une efficacité démontrée chez l'adulte.

Les catastrophes thérapeutiques marquées par l'utilisation de la sulphanilamide dans les années 30 et de la thalidomide dans les années $60,{ }^{[8]}$ auxquelles s'ajoutent de nombreux autres exemples d'effets délétères observés chez l'enfant sans être prévisibles à partir de l'expérience adulte, illustrent la susceptibilité particulière des enfants aux effets indésirables des médicaments. ${ }^{[9]}$ Ce risque est plus marqué en pédiatrie, en raison de l'absence d'évaluation spécifique, ${ }^{[10]}$ du mésusage parental, et par le reconditionnement inadapté des formes pharmaceutiques adultes.

Une méta-analyse de 17 études d'observation d'enfants hospitalisés a permis d'estimer le taux d'effets indésirables à $9,5 \%$ (intervalle de confiance : 6,8-12,2), dont 12,3\% (8,4-16,2) d'effets indésirables graves. ${ }^{[11]}$ Qui plus est, $40 \%$ des accidents à l'origine d'une hospitalisation étaient jugés potentiellement mortels. Rappelons qu'aux Etats-Unis d'Amérique, chaque année, 100000 décès sont imputables aux médicaments chez l'adulte. ${ }^{[12]}$

- Contrairement à une affirmation courante, il n'y a aucune légitimité à considérer que l'éthique de la recherche clinique chez l'enfant est différente de celle chez l'adulte. Le recours à la randomisation repose sur la même clause d'incertitude, et l'utilisation du placebo repose sur la même nécessité de maîtriser les facteurs de confusion.

- Les prescriptions hors AMM, qui restent la situation la plus fréquente, peuvent être considérées comme des expérimentations sauvages et stériles.

\section{La modélisation de population en pédiatrie}

Les projets de modélisation et de simulation débutent par la construction d'un modèle. Dans la plupart des cas un modèle de population est mis en œuvre. ${ }^{[13]}$ Habituellement, ce modèle de population comporte :[14]

- Une sous-unité structurelle qui représente par des équations mathématiques les observations issues des études préalables et les relations identifiées. Cette sous-unité peut être descriptive ou explicative. Par exemple, le modèle de régression logistique pour un critère binaire (résultat succès/échec) représente un modèle descriptif. La description de la clairance hépatique des médicaments chez les jeunes enfants, comme une fonction intégrant le degré de maturation des enzymes hépatiques mesurées in vitro $\left(\mathrm{V}_{\max }\right.$ [vitesse maximale de la réaction enzymatique] et $\mathrm{K}_{\mathrm{M}}$ [constante de Michaelis]), combiné à la fixation protéique et au flux sanguin hépatique, représente un modèle mécanistique. ${ }^{[15,16]}$ Les modèles mécanistiques, ou explicatifs, tentent d'introduire la causalité de l'observation. Les modèles explicatifs permettent quelques extrapolations, alors que les modèles descriptifs ne sont en général pas adaptés à cet objectif. Dans la sous-unité structurelle, les observations sont représentées 
qualitativement, ou condensées quantitativement sous forme des paramètres moyens de population. Les modèles pharmacocinétiques (par exemple à un ou deux compartiments avec une absorption du premier ordre) ou pharmacodynamiques (comme le modèle sigmoïde du $\mathrm{E}_{\max }$ [effet maximum] $)^{[17]}$ ou de la progression de la maladie (comme la dynamique virale $)^{[18]}$ sont des exemples de sous-unités structurelles.

- Une sous-unité statistique qui décrit la variabilité des paramètres dans la population, comme la variabilité interindividus, intra-individu ou inter-occasions. Cette sous-unité comporte aussi habituellement une information quantitative sur la variabilité résiduelle (erreur résiduelle) et sur l'incertitude des paramètres structurels et aléatoires. La distribution normale ou log-normale des paramètres dans la population, l'erreur résiduelle additive ou proportionnelle, et la matrice des variances et covariances des paramètres estimés du modèle constituent des exemples de sous-unités statistiques.

- Une sous-unité des covariables, qui met en relation les paramètres de la population avec les caractéristiques démographiques ou pathologiques telles que l'âge, le poids, le sexe ou un score à l'état de base. ${ }^{[19]}$ L'équation de CockroftGault pour la clairance rénale et la charge virale initiales pour la progression de l'hépatite $\mathrm{C}$ sont des exemples de sous-unités des covariables.

Dans la construction d'un modèle de population, les paramètres du modèle sont généralement estimés par une analyse à effets mixtes. Cette méthode d'analyse permet l'estimation simultanée des paramètres des sous-unités structurelle, statistique et des covariables. Cela permet notamment de conserver les relations fonctionnelles entre les sous-unités composant le modèle, et autorise des simulations cohérentes à partir du modèle.

\section{La modélisation intégrant les connaissances a priori}

La modélisation est un processus permettant l'utilisation de connaissances a priori. Le plus souvent, l'information a priori peut aider à analyser des données nouvelles. L'information disponible a priori sur le médicament, et qui concerne la structure, les aspects statistiques ou les covariables peut être intégrée dans un modèle. L'avantage en est que cette information n'a pas à être générée une fois de plus lors de la planification de l'expérience. Par exemple, pour une étude pharmacocinétique chez l'enfant, l'information a priori peut être représentée par (i) la sous-unité structurelle du modèle pharmacocinétique développé chez l'adulte ; (ii) la sous-unité des covariables décrivant la relation entre la clairance hépatique et/ou rénale avec le poids corporel, ou le processus de maturation dépendant de l'âge chronologique ; (iii) le modèle statistique décrivant la variabilité interindividuelle des paramètres et l'erreur résiduelle attendue pour les observations dans la population d'étude. Cette information a priori peut être issue d'études préalables consacrées au médicament lui-même ou à un médicament présentant des caractéristiques pharmacocinétiques, pharmacodynamiques et/ou thérapeutiques similaires. Elle peut aussi être disponible à partir d'études publiées, concernant par exemple la maturation et le fonctionnement normal ou pathologique des systèmes impliqués. Quel que soit le but dans lequel cette information $a$ priori est utilisée (analyse des données, ou optimisation du plan expérimental), elle contribuera le plus souvent à une meilleure identification des facteurs de risque et à la réduction de l'incertitude autour des prédictions ou des décisions. En fonction du degré de pertinence de l'information disponible a priori sur les médicaments étudiés et des objectifs poursuivis, l'étude prévue peut être planifiée comme explicative ou confirmative.[20] L'utilisation de connaissances a priori est d'une grande importance stratégique en pédiatrie, en raison du nombre généralement limité de patients et d'observations par étude.

L'utilisation de connaissances a priori sur les processus de maturation de la clairance rénale chez le nourrisson a pu être illustrée lors de l'optimisation du plan expérimental d'un médicament antiviral. ${ }^{[21]}$ Elle a conduit à l'ajustement des temps de prélèvement sur l'âge gestationnel. Le partage d'information et le concept de mise en connexion des mondes adultes et pédiatriques ont pu être illustrés aussi à l'occasion de la construction d'un modèle pharmacocinétique de population pour la ciclosporine en pédiatrie. Dans cet exemple, l'estimation précise de certains paramètres du modèle et des covariables d'effets chez l'enfant a été rendue possible par la disponibilité des informations chez l'adulte.

Très fréquemment, le recours à des connaissances a priori provenant d'expériences différentes, comme l'utilisation de données adultes pour l'extrapolation à l'enfant, comporte un risque significatif, celui de propager l'erreur depuis l'un des modèles (données observées) jusqu'à l'autre (dépourvu de ces observations). Bien que cette pratique puisse être aisément justifiée à l'intérieur d'une même population, pour un même objectif et un même plan expérimental, elle ne doit pas être systématiquement recommandée dans les autres cas, et alors toujours utilisée avec une grande prudence.

\section{La certification du modèle}

Un modèle constitue toujours une représentation simplifiée d'une réalité complexe, et comme tel, il ne peut être considéré comme validé. Par contre, il peut être « certifié », en relation avec certains objectifs. Ainsi, dans le mode estimation, un modèle peut 
être évalué par son ajustement aux données, la précision des paramètres estimés, les distributions des paramètres individuels et de l'erreur résiduelle, et par l'adéquation de l'influence des covariables. Dans le mode simulation, un modèle peut être certifié par ses performances prédictives (interpolation et/ou extrapolation). Les métriques utilisées lors de l'évaluation sont d'ordinaire directement pertinentes pour l'utilisation clinique, et indépendantes du modèle. Ainsi, pour les modèles pharmacocinétiques, les métriques peuvent être la $\mathrm{C}_{\max }$ (concentration maximale), la $\mathrm{C}_{\min }$ (concentration minimale), le temps passé au-dessus du seuil de concentration ou l'ASC $\tau$ (aire sous la courbe $\tau$ ) ; pour le modèle de la maladie, les métriques peuvent être le critère de jugement primaire ou secondaire d'un essai clinique d'efficacité. La certification des performances prédictives d'un modèle peut être obtenue à partir des données utilisées lors de la construction du modèle (p. ex., par partition des données en deux jeux, l'un pour la construction du modèle, l'autre pour sa certification, ou en appliquant une procédure de ré-échantillonnage comme le bootstrap), à partir de données anciennes et/ou nouvelles. Idéalement, un modèle doit passer avec succès les épreuves de certification concernant la modélisation et la simulation, avant d'être utilisé dans les décisions cliniques comme le choix de la dose, l' adaptation de la dose, ou l'optimisation du plan expérimental.

\section{Simulation d'essais cliniques}

Lorsqu'un modèle validé est disponible, des simulations peuvent être réalisées. Au-delà des caractéristiques du modèle, de nombreux aspects de l'essai clinique peuvent avoir un impact sur les résultats, comme le plan expérimental (parallèle, croisé, séquentiel, adaptatif, etc.), le nombre et les caractéristiques des patients, les doses et le schéma d'adaptation des doses, le nombre et la périodicité des examens. En outre, il est possible qu'à chaque scénario une méthode ou un plan d'analyse spécifique corresponde. La complexité de l'éventail des possibilités pour la réalisation de l'essai clinique devient telle qu'il est impossible en pratique de se faire une idée de l'impact sur les résultats attendus sans recourir à la simulation. Par analogie avec les simulateurs de vol, des simulateurs d'essais cliniques ont été mis en œuvre pour aider les concepteurs d'essais cliniques à sélectionner le plan expérimental optimal, en fonction du résultat de l'ensemble des combinaisons possibles. ${ }^{[22,23]}$ Cette technologie peut être comprise comme une façon de réduire le risque de mettre en œuvre des essais non conclusifs. Elle ne peut certes jamais remplacer l'étude elle-même. Elle peut optimiser le plan expérimental et/ou mettre en évidence ses limitations. A partir des conclusions des simulations d'essais, le plan expérimental peut être ajusté, optimisé, ou dans certains cas le projet d'essai peut être abandonné.

\section{Optimisation du plan expérimental par la modélisation et la simulation (M\&S) d'essais}

En raison des contraintes éthiques et pratiques, les essais cliniques ne doivent être réalisés en pédiatrie qu'avec parcimonie. Toutes les précautions doivent être prises pour assurer que chaque nouvel examen individuel soit réalisé avec le plus haut niveau d'information possible. Cela soulève la question du plan expérimental optimal des essais pédiatriques. La modélisation et la simulation peuvent être utiles dans ce contexte particulier : la construction des sous-unités structurelle, statistique, et des covariables permet l'identification des composants vis-à-vis desquels le résultat de l'essai est le plus sensible. Réalisée par anticipation avec les informations disponibles a priori, la modélisation permet d'adapter le plan expérimental de l'essai en projet à l'information pertinente, qualitative et quantitative. En voici quelques exemples :

- La maturation des enzymes du métabolisme hépatique ou de la filtration glomérulaire chez le nourrisson peut être modélisée et utilisée pour déterminer leur impact quantitatif sur la règle d'adaptation de la posologie, la planification des prélèvements, la durée de l'observation et l'éventail des âges étudiés.

- La modélisation de la numération des polynucléaires neutrophiles en fonction du temps chez l'enfant traité par chimiothérapie a conduit à proposer un paramètre de sécurité basé sur la pharmacodynamie, et utilisable pour l'optimisation du rapport bénéfice/risque dans les essais cliniques planifiés.

- La variabilité inter-individuelle des paramètres cliniques comme la clairance, le $\mathrm{E}_{\max }$, l'EC $\mathrm{E}_{50}$ (concentration d'efficacité médiane) obtenus à partir de résultats de modélisation publiés, ont pu être utilisés pour affiner l'estimation des effectifs nécessaires à un projet d'essai.

- L'erreur résiduelle liée à des contraintes spécifiques a pu être détectée et réduite par un meilleur contrôle de ces contraintes ou par l'adaptation des examens à ces contraintes.

- Les simulations d'essais cliniques à partir de modèles validés permettent l'évaluation et l'optimisation du plan expérimental. Les résultats peuvent être simulés et analysés suivant des scénarios combinant des variations des doses, des temps d'administration, du nombre et des temps d'examen, du nombre et des caractéristiques des patients, de l'incertitude des paramètres, et des hypothèses du modèle. Avec cette approche, les plans d'expérience et les méthodes d'analyse peuvent tout à la fois être affinés et le meilleur scénario peut être sélectionné.

Cependant, la modélisation a certaines limitations :

- Un exemple moins positif mais représentant un cas d'école intéressant est apporté par le défaut non attendu de diffusion systémique efficace d'une formulation nasale pédiatrique. L'extrapolation directe des données adultes à la situation 
pédiatrique n'avait pas pris en compte que la surface de la muqueuse nasale était en proportion significativement réduite par rapport au poids corporel, chez l'enfant relativement à l'adulte.

Quelques applications peuvent être présentées et discutées à titre d'exemples :

- Lors d'études de pharmacocinétique chez l'enfant, le choix des méthodes d'analyse telles que l'analyse non compartimentale, la modélisation par effets mixtes non linéaires, ou une analyse Bayesienne ${ }^{[24,25]}$ peut interférer très significativement avec le plan de l'essai. Ainsi, des résultats très différents sur l'influence de l'âge post-natal ont pu être obtenus selon que la simulation recourait à une approche populationnelle ou pharmacocinétique classique, pour prévoir l'effet du cisapride. Réciproquement, les contraintes pratiques du plan d'expérience, comme le nombre et le temps des prélèvements, le nombre de sujets pour chaque catégorie peut limiter le choix des méthodes d'analyse. Cette situation a pu être illustrée dans le cas d'un médicament antiviral pour lequel une méthode d'estimation empirique Bayesienne a été adoptée, permettant un ajustement du nombre et du temps des prélèvements.

- Dans les essais de recherche d'éventail de doses, les performances d'un plan d'expérience adaptatif pour la sélection de la dose juste efficace chez l'enfant ont pu être comparées à celles d'un plan séquentiel ou d'un plan classique de doses prédéfinies. L'avantage apparent de la méthode de réévaluation continue recourant à une approche Bayesienne a été illustré dans un essai pour déterminer la dose minimale efficace d'ibuprofène lors de la persistance du canal artériel chez des nouveaux-nés prématurés. ${ }^{[26]}$ Dans cet essai contre placebo, le critère prédéfini de succès (dose associée à une fermeture du canal de $80 \%$ et $50 \%$ chez les nouveaux-nés d'âge gestationnel de 27 à 29 semaines et de moins de 27 semaines, respectivement) a pu être atteint avec la précision requise, après l'inclusion de 20 patients seulement.

- A la fin d'un essai, il n'est pas rare d'observer que l'objectif principal n'est pas atteint de façon indiscutable. Grâce à la modélisation et la simulation, de telles situations peuvent être anticipées et une décision correctement informée peut être prise. ${ }^{[27]}$ La performance du plan d'expérience à réunir les contraintes prédéfinies peut être évaluée quantitativement, et la décision de réaliser l'essai peut être prise ou non avec ses limitations intrinsèques bien précisées. Cette discussion a pu être illustrée à partir de l'exemple du midazolam par voie rectale pour la sédation des enfants au cours d'un cathétérisme cardiaque : la simulation a montré que la dose active était supérieure à ce que les données sur la sécurité permettaient d'admettre, et la formulation abandonnée. ${ }^{[28]}$

\section{Recommandations, formation et réseau professionnel pour la M\&S}

Des recommandations de bonnes pratiques de $M \& S$ sont déjà disponibles, comme celles mises à disposition par le CDDS (Centre for Drug Development Science).

L'utilisation adéquate de la M\&S nécessite des compétences professionnelles spécifiques. De même que pour la pharmacocinétique de population, ${ }^{[29]}$ ainsi que les guides pour les relations exposition-effet de la FDA (US Food and Drug Administration), ${ }^{[30]}$ la modélisation ne doit pas s'affranchir des étapes de validation, en évaluant la cohérence interne du modèle, ainsi que son agrément avec les données externes, c'est-à-dire les faits et les autre modèles.

Les participants de la $6^{\text {ème }}$ Table Ronde de Giens 2004 recommandent : (i) que le recours à la modélisation soit systématiquement envisagé et, s'il est décidé, que la modélisation soit conduite, interprétée, validée et appliquée de façon adaptée ; (ii) dans la mesure où la modélisation et la simulation sont par nature des approches multidisciplinaires, que toutes les compétences professionnelles requises soient mises en œuvre, à tous les stades, y compris le processus d'approbation technique du rapport de $M \& S$; (iii) que toutes les données requises pour une modélisation optimale soient accessibles, grâce à un partenariat approprié entre industriels, universitaires, régulateurs et autorités de santé ; (iv) que les cliniciens soient systématiquement conscients des apports potentiels de la M\&S, et qu'ils aient l'expérience au moins de l'interprétation des résultats de M\&S ; (v) que les spécialistes du domaine développent des algorithmes ou arbres de décision pour aider les non-spécialistes à décider quand il est nécessaire de recourir à la $M \& S$; (vi) que les universités proposent aux professionnels du médicament des formations appropriées dans le domaine des plans d'expérience, de la conduite des essais cliniques, de l'analyse des données pharmacocinétiques et pharmacodynamiques, de la modélisation et de la simulation.

\section{Conclusions}

Les règles évoluent lentement dans la bonne direction pour la promotion de la recherche clinique chez l'enfant, ce qui permettra à terme d'assurer une offre de soins de meilleure qualité à cette population particulièrement fragile. La modélisation et la simulation apparaissent des outils essentiels à l'atteinte des objectifs finaux de cette évolution. L'utilisation systématique et judicieuse de ces outils sera facilitée par :

- l'éducation et la formation ;

- la disponibilité de modèles conviviaux en accès libre par Internet pour les praticiens ;

- et un accès libre aux bases de données pour l'ensemble des populations de petits effectifs. 


\section{Participants}

D. Armengaud (CH, Saint-Germain en Laye), I. Bost (Grenoble), A. Carpentier (LEEM, Paris), C. Caulin (Hôpital Lariboisière, Paris), C. Crépin (EMF Consulting, Aix en Provence), E.-M. Fuseau (EMF Consulting, Aix en Provence), M. Gerberg (Knoll France, Chilly-Mazarin), C. Grosskopf (Roche, Neuilly-sur-Seine), F. Gueyffier (Centre d'Investigation Clinique, Lyon), N Hoog Labouret (Afssaps, Saint-Denis), P. Jacqmin (Sté Exprimo, Beerse, Belgique), C. Laveille (IRIS, Courbevoie), C. Le Gellec (Hôpital Bretonneau, Tours), P. Marquet (CH Dupuytren, Limoges), A. Ouslimani (DRRC, AP-HP, Paris), G. Pons (Hôpital Saint-Vincent de Paul, Paris), U. Simeoni (La Timone, Marseille), N. Simon (Faculté de Médecine, Marseille), B. Tranchand (Faculté de Médecine, Lyon), J.-M. Treluyer (Hôpital Saint-Vincent de Paul, Paris).

\section{Références}

1. Section 111: Pediatric studies of drugs. Food and Drug Administration Moderization Act of 1997 [online]. Available from URL: http://www.fda.gov/cder/pediatric [Accessed 2005 Aug 8]

2. Pediatric Rule 1998 [online]. Available from URL: http://www.fda.gov/cder/pediatric [Accessed 2005 Aug 8]

3. Public law 107-109, Best Pharmaceuticals for children Act 2001 [online]. Available from URL: http://www.fda.gov/cder/pediatric [Accessed 2005 Aug 8]

4. Pediatric Research Equity Act of 2003 [online]. Available from URL http://www.fda.gov/opacom/laws/prea.html [Accessed 2005 Aug 8]

5. Proposal for a Regulation of the Council and of the Parliament on Medicina Products for Paediatric Use [online]. Available from URL: http://pharmacos.eudra.org/F2/Paediatrics/Index.htm [Accessed 2005 Jul 6]

6. Chalumeau M, Treluyer JM, Salanave B, et al. Off label and unlicensed drug use among French office based paediatricians. Arch Dis Child 2000; 83 (6): 502-5

7. Fontan JE, Combeau D, Brion F. Pediatric drug preparations in French hospitals: Pediatric Group of the French Clinical Pharmacy Society. Arch Pediatr 2000; 7 (8): 825-32

8. Botting J. The history of thalidomide. Drug News Perspect 2002; 15 (9): 604-11

9. Conroy S, Choonara I, Impicciatore P, et al. Survey of unlicensed and off label drug use in paediatric wards in European countries. European Network for Drug Investigation in Children. BMJ 2000; 320 (7227): 79-82

10. Impicciatore $\mathrm{P}$, Pandolfini $\mathrm{C}$, Bonati $\mathrm{M}$. Database could give children safer medicines [letter]. Nature 2000; 405 (6789): 882

11. Impicciatore $\mathrm{P}, \mathrm{Choonara} \mathrm{I}, \mathrm{Clarkson} \mathrm{A}$, et al. Incidence of adverse drug reactions in paediatric in/out-patients: a systematic review and meta-analysis of prospective studies. Br J Clin Pharmacol 2001; 52 (1): 77-83

12. Lazarou J, Pomeranz BH, Corey PN. Incidence of adverse drug reactions in hospitalized patients: a meta-analysis of prospective studies. JAMA 1998; 279 (15): 1200-5

13. Piotrovsky V. Population pharmacodynamic and pharmacokinetic modelling via mixed effects. Current Opin Drug Discov Devel 2000; 3 (3): 314-30

14. Sheiner L, Steimer JL. Pharmacokinetic/pharmacodynamic modelling in drug development. Annu Rev Pharmacol Toxicol 2000; 40: 67-95

15. Johnson T, Tanner M, Taylor C, et al. Enterocytic CYP3A4 in a paediatric population: developmental changes and the effects of celiac disease and cystic fibrosis. Br J Clin Pharmacol 2001; 51: 451-60
16. Johnson $\mathrm{T}$. The development of drug metabolizing enzymes and their influence on the susceptibility to adverse drug reactions in children. Toxicology 2003; 192: $37-48$

17. Reid AW, Anderson BJ, Futter ME. Relationship of muscle strength to potassium concentration in a hypokalaemic infant. Anaesth Intensive Care 1997; 25 (5): $525-7$

18. Funck GA, Fischer M, Joos B. Quantification of in vivo replicative capacity of HIV-1 in different compartments of infected cells. J Acquir Immune Defic Syndr 2001; 26 (5): 397-404

19. Mandema J, Verrota D, Sheiner L. Pharmacometrics: building population pharmacokinetic-pharmacodynamic models: I. Models for covariate effects. J Pharmacokinet Biopharm 1992; 20 (5): 511-28

20. Sheiner L. Learning versus confirming in clinical drug development. Clin Pharmacol Ther 1997; 61 (3): 275-91

21. Tod M, Lokiec F, Bidault R, et al. Pharmacokinetics of oral acyclovir in neonates and in infants: a population analysis. Antimicrob Agents Chemother 2001; 45 (1): $150-7$

22. Holford N, Kimko H, Monteleone J, et al. Simulation of clinical trials. Annu Rev Pharmacol Toxicol 2000; 40: 209-34

23. Girard P, Cucherat M, Guez D. Simulation des essais cliniques dans le développement des médicaments. Therapie 2004; 59 (3): 287-304

24. Patoux A, Bleyzac N, Boddy A, et al. Comparison of nonlinear mixed-effect and non-parametric expectation maximisation modelling for Bayesian estimation of carboplatin clearance in children. Eur J Clin Pharmacol 2001; 57: 297-303

25. Rubie H, Doz F, Vassal G, et al. Individual dosing of carbolplatin based on drug monitoring in children receiving high-dose chemotherapy. Eur J Cancer 2003; 39: $1433-8$

26. Desfrere L, Zohar S, Morville P, et al. Dose-finding study of ibuprofen in patent ductus arteriosus using the continual reassessment method. J Clin Pharm Ther 2005; 30 (2): 121-32

27. Gieschke R, Steimer JL. Pharmacometrics: modelling and simulation tools to improve decision making in clinical drug development. Eur J Drug Metab Pharmacokinet 2000; 25 (1): 49-58

28. Fabre E, Chevret S, Piechaud JF, et al. An approach for dose finding of drugs in infants: sedation by midazolam studied using the continual reassessment method. Br J Clin Pharmacol 1998; 46: 395-401

29. Guidance for industry: population pharmacokinetics, February 1999 [online]. Bethesda (MD): US Department of Health and Human Services, Food and Drug Administration, Center for Drug Evaluation and Research (CDER), Center for Biologics Evaluation and Research (CBER). Available from URL: http://www.fda.gov/cder/guidance/1852fnl.pdf [Accessed 2004 Dec 1]

30. Exposure-response relationships: study design, data analysis, and regulatory applications. 1998; 46: 395-401. Bethesda (MD): US Department of Health and Human Services, Food and Drug Administration, Center for Drug Evaluation and Research (CDER) Center for Biologics Evaluation and Research (CBER) April 2003 [online]. Available from URL: http://www.fda.gov/ohrms/dockets/ac/99/backgrd/3457b2a.pdf [Accessed 2004 Dec 1]

Correspondance et offprints : François Gueyffier, Centre d'Investigation Clinique, Hôpital Louis Pradel, 28 avenue du Doyen Lépine, 69500 Bron, France.

E-mail : francois.gueyffier@chu-lyon.fr 